\title{
Identification of highly expressed, soluble proteins using an improved, high-throughput pooled ORF expression technology
}

\author{
Timothy Waybright1, William K. Gillette2, Dominic Esposito², Robert M. Stephens³, David A. \\ Lucas $^{1}$, James L. Hartley², and Timothy D. Veenstra ${ }^{1}$
}

BioTechniques 45:307-315 (September 2008)

doi 10.2144/000112916

\begin{abstract}
This article describes an improved pooled open reading frame (ORF) expression technology (POET) that uses recombinational cloning and solution-based tandem mass spectrometry (MS/MS) to identify ORFs that yield high levels of soluble, purified protein when expressed in Escherichia coli. Using this method, three identical pools of 512 human ORFs were subcloned, purified, and transfected into three separate E. coli cultures. After bulk expression and purification, the proteins from the three separate pools were digested into tryptic peptides. Each of these samples was subsequently analyzed in triplicate using reversed-phase high-performance liquid chromatography $(L C)$ coupled directly online with MS/MS. The abundance of each protein was determined by calculating the average exponentially modified protein abundance index (emPAI) of each protein across the three protein pools. Human proteins that consistently gave high emPAI values were subjected to small-scale expression and purification. These clones showed high levels of expression of soluble protein. Conversely, proteins that were not observed by LC-MS/MS did not show any detectable soluble expression in small-scale validation studies. Using this improved POET method allows the expression characteristics of hundreds of proteins to be quickly determined in a single experiment.
\end{abstract}

\section{INTRODUCTION}

While sequencing of the human genome has obviously been a major benefit to genomics, it has also opened a number of research opportunities in proteomics: thousands of open reading frames (ORFs) can now be cloned and expressed as proteins (1). Significant efforts have been devoted to converting these clones into soluble, purified proteins that can be used for structural studies $(2,3)$. There are presently a number of structural proteomics initiatives around the world that use X-ray crystallography or nuclear magnetic resonance (NMR) spectroscopy attempting to determine the three-dimensional (3-D) structures of one or more proteins from each protein family with the goal of predicting their function $(4,5)$. While computational methods of predicting a protein's 3-D structure based on its sequence have dramatically improved $(6,7)$, a large number of proteins remain whose sequences do not show enough homology to known structures to make structure predictions successful (8). In these cases, sufficient amounts of the protein must be expressed and purified prior to acquisition of the requisite $\mathrm{X}$-ray crystallography or NMR spectroscopy data. Arguably the rate-limiting step in structural biology is in the production of large amounts of proteins with high enough quality for structural studies. Current figures estimate that roughly half of the proteins represented within the human genome are expected to be difficult to express or solubilize (9-11).

Another area in which purified proteins arising from the predicted ORFs in the human genome would have a tremendous impact is in the generation of affinity reagents $(12,13)$.
Array platforms for genomic analysis are commonplace; however, their use in proteomics has not been able to keep pace due to the lack of high affinity reagents for large numbers of proteins found in the human proteome. While antibodies can be generated against synthetic peptides, the availability of the native protein generally produces reagents with greater specificity and utility.

One of the bottlenecks in acquiring a sufficient amount of purified protein with which to conduct structural studies or generate affinity reagents is obtaining high levels of soluble expression (9). Determining the expression characteristics of a cloned ORF in a specific system and under a specific set of conditions is usually a matter of trial and error $(14,15)$. The standard approach requires cloning individual ORFs into an expression vector, which is introduced into an 


\section{Research Reports}

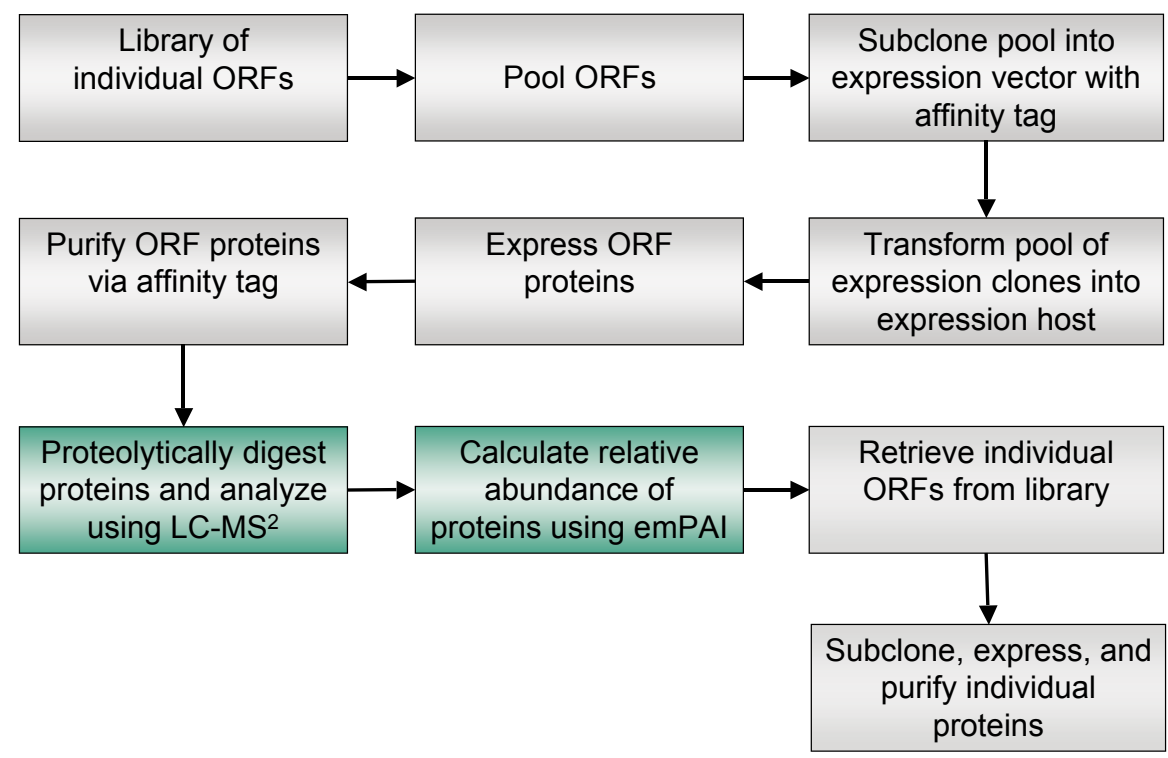

Figure 1. Improved pooled open reading frame (ORF) expression technology (POET) method. The green boxes highlight improcxvements made to the previous method that dramatically increase the throughput in recognizing clones that efficiently express using POET.

expression host, and then expressed in separate cultures under a number of different conditions such as induction, time, and temperature. Those cultures that show positive expression are grown and induced at a scaled-up level so that protein purification can be attempted. While automation has helped, the logistics and costs of this high-risk strategy are enormous when one considers the expression of hundreds or thousands of ORFs.

We previously described a method termed pooled ORF expression technology (POET) that combines recombinational cloning and collections of sequenced ORFs with proteomic methods [two-dimensional polyacrylamide gel electrophoresis (2D-PAGE) and tandem mass spectrometry (MS/MS)] to predict which ORFs in a pool will yield soluble, purified protein (16). While this method had a high success rate in identifying which ORFs expressed high levels of soluble protein, its overall throughput was low. The bottleneck in the procedure was the need to separate the protein mixture using 2D-PAGE prior to MS/MS identification of the hundreds of visualized proteins spots. In addition, Escherichia coli proteins cannot be distinguished from those expressed by the human ORFs using 2D-PAGE, resulting in a necessarily high percentage of the experimental time being devoted to identification of host proteins. As described below, we have dramatically improved the throughput of POET by obviating the need to quantify the expressed proteins through visualization of a $2 \mathrm{D}-\mathrm{PAGE}$ gel. In this improved approach, the relative abundance of the expressed proteins is determined by calculating their exponentially modified protein abundance index (emPAI) (17) using data acquired by direct reversed-phase high-performance liquid chromatography-MS/MS (LC-MS/MS) analysis of the expressed protein pool. As with the previous study, a high percentage of ORFs identified in this experiment yielded expressed, soluble, purified proteins in agreement with POET predictions. The consistency of the data in the nine replicates and the simplicity of the method represent a significant improvement over the earlier POET protocol.

\section{MATERIALS AND METHODS}

\section{ORF Pool}

The Homo sapiens ORFeome version 1 has been described previously (18). The DNA concentrations of the 7864 Gateway entry clones used in this experiment were determined by PicoGreen (Molecular Probes, Invitrogen, Carlsbad, CA, USA) fluorescence and used to calculate the molar concentration of each plasmid (based on the size of each ORF and the size of the pDONR223 backbone), which ranged from 0 to $23.1 \mathrm{nM}$. All wells containing $<0.15 \mathrm{nM}$ plasmid concentration were omitted, leaving 7352 ORFs. These clones were further subdivided into two classes ("known" and "unknown") based on the following criteria: "unknowns" must (i) have no predicted transmembrane domains, (ii) have no predicted secretion signal sequence, and (iii) have no structure of themselves or any homolog in the Protein Data Bank. We decided on $30 \%$ as the cutoff for homology to produce a pool of proteins of interest to researchers that were reasonable likely to be purifiable. This "unknown" pool contained 3279 clones. The pool was subdivided further into six subpools of approximately 512 ORFs each, which were set up to contain an even distribution of insert sizes. These pools were designated U1 through U6, and were concentration-normalized by the method previously described for the POET analysis of ORFs obtained from Caenorhabditis elegans (16). The pools were ethanol-precipitated 


\section{Research Reports}

Table 1. Proteins from Sample 1 (Run in Triplicate), with the Highest emPAI Scores Showing Individual Sample emPAI Score

\begin{tabular}{|lccccccc|}
\hline Protein & $\begin{array}{c}\text { Theoretical } \\
\text { Peptide Count }\end{array}$ & \multicolumn{2}{c|}{$\begin{array}{c}\text { Peptide } \\
\text { Count }\end{array}$} & \multicolumn{1}{c|}{ emPAI } \\
Scores
\end{tabular}

and dissolved in Tris-EDTA to a final concentration of $5 \mathrm{ng} / \mu \mathrm{L}$.

\section{Protein Expression}

A single pool containing 512 ORFs [as Gateway attL entry clones (6)] was subcloned into pDest527 (T7 promoter, amino-terminal $\mathrm{His}_{6}$ fusion) with LR Clonase (Invitrogen) according to the manufacturer's instructions, except that the reaction was allowed to proceed for $5 \mathrm{~h}$ at $30^{\circ} \mathrm{C}$. Each of the 512 ORFs expressed in this vector contained the sequence MRSGSHHHHHHRSDITSLYKKAG added to its amino end and YPAFLYKVVISLAR added to its carboxyl end due to the lack of the native stop codon. Reaction products were transformed into DH5 $\alpha$ cells (Invitrogen), and $1 \%$ of the SOC expression mixture was plated on ampicillin. The remaining $99 \%$ of the expression mixture was added to 50 mL CircleGrow (QBiogene, Carlsbad, CA, USA) containing ampicillin, and after overnight growth at $37^{\circ} \mathrm{C}$, plasmid DNA was purified (Fast Plasmid; Brinkmann, Westbury, NY, USA). Approximately $100 \mathrm{ng}$ pooled expression plasmids were electroporated into $E$. coli Rosetta (DE3) strain (Novagen, EMD Chemicals, San Diego, CA, USA), which compen- sates for eukaryotic codons that are rare in E. coli. The $1 \mathrm{~mL}$ SOC expression mixture was diluted into $50 \mathrm{~mL}$ CircleGrow (containing $100 \mu \mathrm{g} / \mathrm{mL}$ ampicillin) and grown overnight at $37^{\circ} \mathrm{C}$. The overnight culture was diluted $1: 100$ into $1 \mathrm{~L}$ CircleGrow (containing $100 \mu \mathrm{g} / \mathrm{mL}$ ampicillin), grown at $37^{\circ} \mathrm{C}$ to an $\mathrm{A}_{600}$ of 0.5 , and cooled to $16^{\circ} \mathrm{C}$, at which time protein expression was induced by adding isopropyl 1-thio- $\beta$-Dgalactopyranoside to a final concentration of $0.5 \mathrm{mM}$. After $16 \mathrm{~h}$ at $16^{\circ} \mathrm{C}$, cells were harvested and frozen at $-80^{\circ} \mathrm{C}$. For individual expression of positive and negative clones, individual ORFs were subcloned by LR recombination into pDest527, and expression clones were individually transformed into $E$. coli Rosetta (DE3) cells and induced under the same conditions as above. Whole-cell samples for gel electrophoresis were generated by centrifugation of 0.05 optical density (OD) units of induced cells, followed by freeze-thaw at $-80^{\circ} \mathrm{C}$, treatment with $1 \mathrm{U}$ benzonase nuclease at $37^{\circ} \mathrm{C}$ for $15 \mathrm{~min}$, and addition of standard sodium dodecyl sulfate PAGE (SDS-PAGE) buffers and tris(2-carboxyethyl)phosphine (TCEP). Soluble fractions were generated by a mild detergent lysis of 1 OD units of induced cells using the Readypreps procedure (Epicentre Biotechnologies, Madison, WI, USA). All gel samples were analyzed by SDS-PAGE using 4\%-20\% Criterion gels (Bio-Rad Laboratories, Hercules, CA, USA).

\section{Protein Purification}

Proteins were purified using immobilized metal affinity chroma-

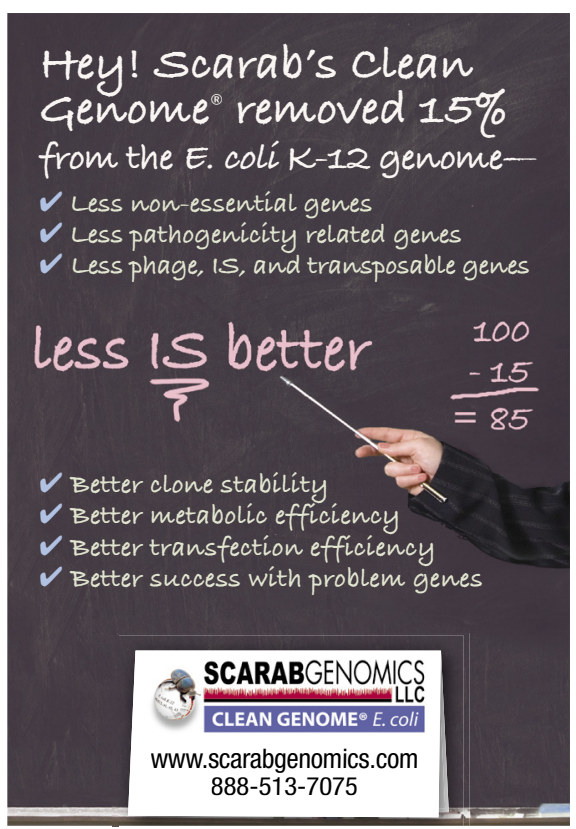




\section{BioTechniques}

Connecting. Informing. Advancing.

For 25 Years.

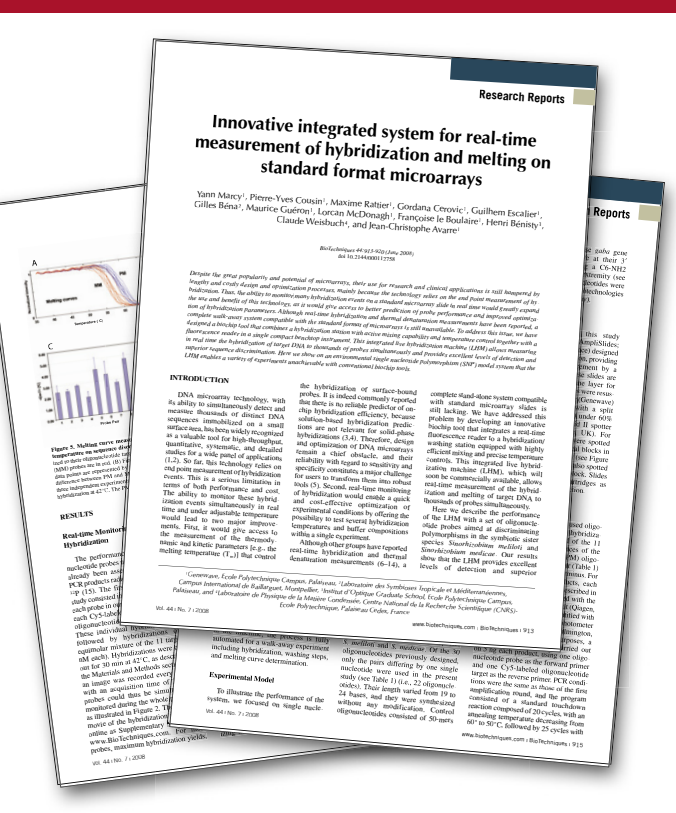

\section{Reprints}

\section{Author Reprints}

Make the most of your hard work by ordering reprints of your article published in BioTechniques. Reprints are an inexpensive and easy way to distribute your findings to students and colleagues alike.

\section{Corporate Reprints}

Leverage BioTechniques, the most powerful brand in the market. Reprints help support your sales effort by utilizing articles that spotlight your brand/products to educate customers at meetings and industry trade events.

To order:

reprints@biotechniques.com

More info:

www.biotechniques.com/reprints 212-520-2714

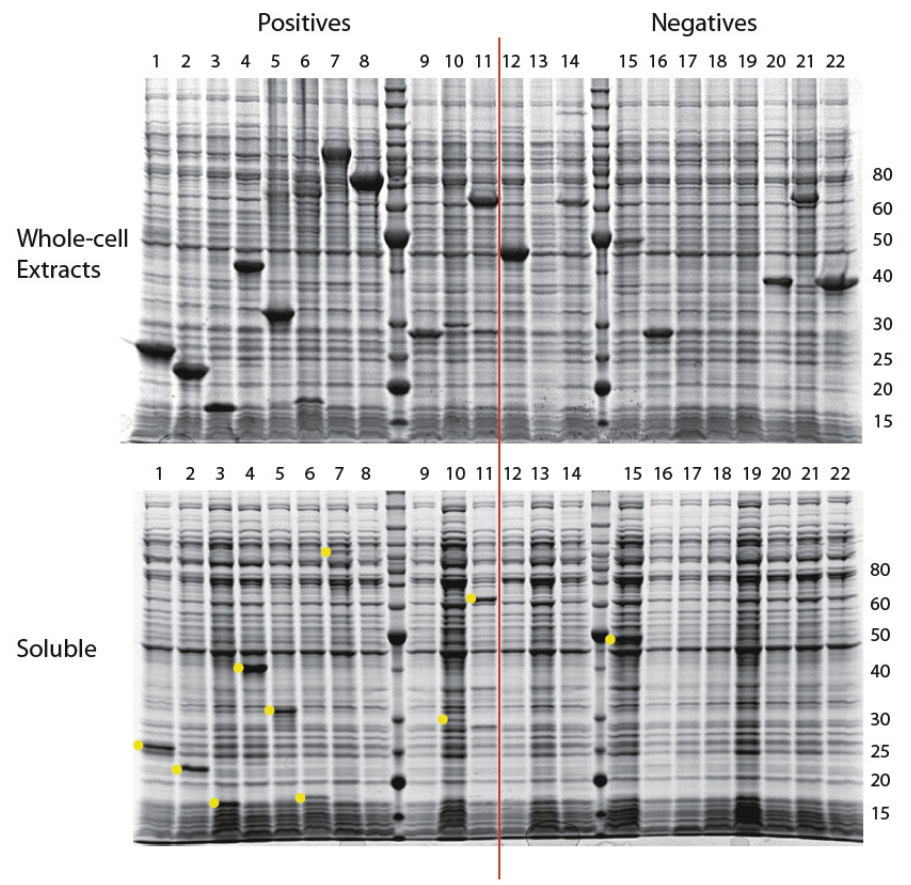

Figure 2. SDS-PAGE analysis of 11 pooled open reading frame (ORF) expression technology (POET) positives [high exponential modified protein abundance (emPAI) scores, lanes 1-11] and negatives (emPAI scores equal to zero, lanes 12-22) from whole-cell extracts and soluble supernatants of induced small-scale cultures containing individual clones. The yellow dots indicate the positions of the stained protein band produced from expression of the individual cloned ORF. The numbering of the gel lanes correspond to the ORFs listed in Tables 4 and 5.

tography (IMAC) as described previously (16). E. coli cell pastes were resuspended using two volumes of extraction buffer per gram of wet weight to achieve a final concentration of $20 \mathrm{mM}$ sodium phosphate buffer, $\mathrm{pH} 7.5,100 \mathrm{mM} \mathrm{NaCl}, 5 \mathrm{mM} \mathrm{MgCl}$, $5 \%$ glycerol, $45 \mathrm{mM}$ imidazole, and complete protease inhibitor-EDTA (Roche Applied Science, Indianapolis, IN, USA) at one tablet $/ 50 \mathrm{~mL}$ extract. Cell extracts were treated with $0.5 \mathrm{mg} /$ $\mathrm{mL}$ lysozyme for $30 \mathrm{~min}$ and $1 \mathrm{U} / \mathrm{mL}$ benzonase (Novagen) for an additional $20 \mathrm{~min}$. Samples were sonicated to lyse the cells, and the lysates was adjusted to $500 \mathrm{mM} \mathrm{NaCl}$. The lysate was centrifuged at $111,000 \times g$ for $30 \mathrm{~min}$, filtered $(0.45 \mu \mathrm{m}$, polyethersulfone membrane), and applied at $0.6 \mathrm{~mL} / \mathrm{min}$ to $1 \mathrm{ml}$ HisTrap columns (Amersham Biosciences, Piscataway, NJ, USA) equilibrated with extraction buffer in $500 \mathrm{mM} \mathrm{NaCl}$ and $45 \mathrm{mM}$ imidazole (binding buffer). The columns were washed with binding buffer until the levels of protein flowing through the column reached base line. Bound proteins were eluted with binding buffer containing $500 \mathrm{mM}$ imidazole into $1 \mathrm{~mL}$ fractions, which were subsequently analyzed using SDS-PAGE. The pools created from the IMAC fractions were precipitated by adding $25 \%(\mathrm{v} / \mathrm{v})$ trichloroacetic acid (TCA) to a final concentration of $6 \%(\mathrm{v} / \mathrm{v})$. After vortex mixing, the samples were incubated on ice for $5 \mathrm{~min}$ followed by centrifugation at $16,100 \times g$ for 10 min. The supernatant was removed, and the pellet incubated with ice-cold acetone for $5 \mathrm{~min}$ on ice followed by centrifugation at $16,100 \times g$ for 5 min. The supernatant was discarded, and the pellet was dried for $2 \mathrm{~min}$ at $70^{\circ} \mathrm{C}$. The pellet was finally dissolved in solubilization buffer ( $8 \mathrm{M}$ urea, $4 \%$ CHAPS, $50 \mathrm{mM}$ Tris, $\mathrm{pH}$ 8.5) at room temperature to a concentration of 20 $\mathrm{mg} / \mathrm{mL}$ and stored in $50 \mu \mathrm{L}$ aliquots at $-80^{\circ} \mathrm{C}$.

\section{Sample Preparation and MS/MS Analysis}

The pooled samples were digested with trypsin using $5 \mathrm{mM}$ dithiothreitol (DTT), $25 \mathrm{mM} \mathrm{NH}_{4} \mathrm{HCO}_{3}$, and $0.5 \mu \mathrm{g}$ 


\section{Research Reports}

sequencing-grade modified trypsin (Promega, Madison, WI, USA). All of the samples were desalted with $\mathrm{C}_{18} \mathrm{Zip}$ Tips (Millipore, Billerica, MA, USA) following the manufacturer's protocols prior to MS analysis. Chromatographic separations of desalted tryptic peptides were conducted using nanocolumns prepared in-house. A $75 \mu \mathrm{m}$ inner diameter $\times 360 \mu \mathrm{m}$ outer diameter $\times 10 \mathrm{~cm}-$ long fused silica capillary column (Polymicro Technologies, Phoenix, AZ, USA) with one end flame-pulled to a fine tip $(\sim 5-$ to $7-\mu \mathrm{m}$ orifice) was slurry-packed with $5 \mu \mathrm{m}$, 300 - $\AA$ pore size $\mathrm{C}_{18}$ stationary phase (Phenomenex, Torrance, CA, USA).

The reversed-phase separations were performed by injecting $5 \mu \mathrm{L}$ sample per analysis. The columns were connected via a stainless steel union to an Agilent 1100 Nanoflow LC system (Agilent Technologies, Palo Alto, CA, USA), which was used to deliver solvents $\mathrm{A}(0.1 \% \mathrm{HCOOH}$ in water $)$ and $\mathrm{B}\left(0.1 \% \mathrm{HCOOH}\right.$ in $\left.\mathrm{CH}_{3} \mathrm{CN}\right)$. After the sample was injected, a 20 min wash with $98 \%$ mobile phase A was used to flush any remaining salts from the sample. Peptide elution was accomplished using a linear gradient of $2 \%$ mobile phase B to $42 \%$ solvent B over $40 \mathrm{~min}$ with a constant flow rate of $250 \mathrm{~nL} / \mathrm{min}$. The column was flushed for 15 min with $98 \%$ mobile phase B and re-equilibrated with $98 \%$ mobile phase A prior to subsequent sample loading.

The nanoflow reversed-phase LC column was coupled online to a LIT mass spectrometer (LTQ; ThermoElectron, Thermo Fisher Scientitic, San Jose, CA, USA) using the manufacturer's nanoelectrospray source with an applied electrospray potential of $1.7 \mathrm{kV}$ and capillary temperature of $160^{\circ} \mathrm{C}$. The LIT mass spectrometer was operated in a datadependent mode in which each full MS scan was followed by five MS/MS scans, where the five most abundant peptide molecular ions detected from the MS scan were dynamically selected for five subsequent MS/MS scans using a normalized collision-induced dissociation (CID) energy of $35 \%$ and a dynamic exclusion of $60 \mathrm{~s}$ to reduce redundant selection of peptides. The CID spectra were analyzed using SEQUEST operating on a Beowulf 18-node parallel virtual machine cluster computer (ThermoElectron, Thermo Fisher Scientific, Waltham, MA, USA) using a combined UniProt nonredundant $E$. coli and human proteome database (containing 43,137 protein sequences) downloaded January 2006 (www.expasy.org). Only peptides with conventional tryptic termini (allowing for up to two internal missed cleavages) possessing delta-correlation scores $\left(\Delta C_{n}\right)>0.08$ and charge state-dependent crosscorrelation $\left(X_{\text {corr }}\right)$ criteria as follows were considered as legitimate identifi-

Table 2. Mean emPAI Scores Obtained from Three Separate POET Experiments

\begin{tabular}{|c|c|c|c|}
\hline Protein & & emPAI Scores & \\
\hline & $\operatorname{Exp} 1$ & $\operatorname{Exp} 2$ & $\operatorname{Exp} 3$ \\
\hline FKBP-type peptidyl-prolyl cis-trans isomerase slyD & $0.730 \pm 0.000$ & $0.804 \pm 0.220$ & $0.670 \pm 0.104$ \\
\hline Protein CGI-38 & $0.535 \pm 0.089$ & $0.363 \pm 0.126$ & $0.469 \pm 0.056$ \\
\hline UPF0235 protein C15orf40 & $0.440 \pm 0.108$ & $0.500 \pm 0.056$ & $0.670 \pm 0.107$ \\
\hline 60 kDa Chaperonin (GroEL) & $0.407 \pm 0.013$ & $0.386 \pm 0.067$ & $0.349 \pm 0.000$ \\
\hline Ribosomal small subunit pseudouridine synthase $A$ & $0.274 \pm 0.031$ & $0.295 \pm 0.110$ & $0.238 \pm 0.000$ \\
\hline Developmental pluripotency associated 4 & $0.271 \pm 0.037$ & $0.292 \pm 0.057$ & $0.239 \pm 0.080$ \\
\hline $26 S$ Proteasome subunit p27 & $0.241 \pm 0.029$ & $0.241 \pm 0.029$ & $0.208 \pm 0.057$ \\
\hline Catabolite gene activator & $0.240 \pm 0.071$ & $0.338 \pm 0.106$ & $0.164 \pm 0.044$ \\
\hline Major outer membrane lipoprotein precursor (OmpF) & $0.233 \pm 0.000$ & $0.151 \pm 0.071$ & $0.237 \pm 0.129$ \\
\hline Optineurin & $0.210 \pm 0.053$ & $0.214 \pm 0.015$ & $0.159 \pm 0.041$ \\
\hline Hypothetical protein MGC4504 & $0.199 \pm 0.000$ & $0.152 \pm 0.041$ & $0.176 \pm 0.041$ \\
\hline Coiled-coil domain-containing protein 16 & $0.197 \pm 0.013$ & $0.182 \pm 0.022$ & $0.125 \pm 0.024$ \\
\hline RNA-binding protein with multiple splicing & $0.180 \pm 0.065$ & $0.076 \pm 0.034$ & $0.057 \pm 0.058$ \\
\hline Drebrin-like protein & $0.178 \pm 0.014$ & $0.257 \pm 0.083$ & $0.161 \pm 0.043$ \\
\hline 30S Ribosomal subunit protein S15 & $0.157 \pm 0.083$ & $0.075 \pm 0.000$ & $0.075 \pm 0.000$ \\
\hline Bifunctional polymyxin resistance arnA protein & $0.136 \pm 0.010$ & $0.114 \pm 0.010$ & $0.077 \pm 0.032$ \\
\hline Hypothetical protein MGC20255 & $0.133 \pm 0.035$ & $0.144 \pm 0.021$ & $0.064 \pm 0.000$ \\
\hline KIAA1143 & $0.129 \pm 0.000$ & $0.129 \pm 0.046$ & $0.056 \pm 0.025$ \\
\hline Sperm surface protein Sp17 & $0.124 \pm 0.033$ & $0.034 \pm 0.030$ & $0.017 \pm 0.030$ \\
\hline
\end{tabular}




\section{Research Reports}

cations: $>1.9$ for +1 charged peptides, $>2.2$ for +2 charged peptides, and $>3.1$ for +3 charged peptides.

\section{Quantitation of Protein Abundance}

The relative abundance of proteins in the POET pooled was calculated using emPAI as described previously (17). In this method, the PAI value for each protein is initially calculated and then converted to a corresponding emPAI score by raising 10 to the power of PAI, then subtracting 1 (10 ${ }^{\text {PAI }} 1$ ). The PAI value is determined by dividing the number of experimentally identified peptide fragments from an MS analysis by the total number of possible observable peptide fragments $\left(\mathrm{N}_{\text {obsvd }} / \mathrm{N}_{\text {obsvbl }}\right)$ for each individual protein. The emPAI index allows a quick and easy estimation of total protein content by normalizing the data to the number of theoretical peptides in a mixture of proteins. The emPAI values were calculated and averaged over three separate LC-MS/ MS analyses of each of the three samples of purified proteins obtained from the expressed ORF pools (nine replicates total).

\section{RESULTS}

We previously developed the POET method as a procedure for finding which ORFs in a pool of hundreds of ORFs can be most efficiently converted, by cloning, expression, and purification, into their corresponding recombinant proteins (16). By combining $n$ ORFs into a single pool, tasks that need to be conducted for each individual clone (transformation, plating, colony picking, culture, induction, lysis, assays of solubility, and purification) are reduced $n$-fold. Where the previous method was lacking was in the need to quantitate the expression levels of the individual ORFs through the visualization of the proteins separated using 2D-PAGE. The throughput of this method was severely compromised by the need to conduct in-gel digestion and MS identification of all of the visualized spots. A significant portion of the spots were identified as host $E$. coli

Table 3. Rank of Proteins by emPAI Value from Three Separated Experiments

\begin{tabular}{|c|c|c|c|}
\hline \multirow[t]{2}{*}{ Protein } & \multicolumn{3}{|c|}{ emPAI Rank } \\
\hline & Exp 1 & $\operatorname{Exp} 2$ & Exp 3 \\
\hline FKBP-type peptidyl-prolyl cis-trans isomerase slyD & 1 & 1 & 2 \\
\hline Chaperone protein DnaK & 2 & 3 & 5 \\
\hline Protein CGI-38 & 3 & 7 & 4 \\
\hline UPF0235 protein C15orf40 & 4 & 2 & 1 \\
\hline 60 kDa Chaperonin (GroEL) & 5 & 6 & 6 \\
\hline Ferric uptake regulation protein & 6 & 5 & 16 \\
\hline Programmed cell death protein 5 & 7 & 4 & 3 \\
\hline Ribosomal small subunit pseudouridine synthase $A$ & 8 & 9 & 8 \\
\hline Developmental pluripotency associated 4 & 9 & 10 & 7 \\
\hline 26S Proteasome subunit p27 & 10 & 12 & 11 \\
\hline Catabolite gene activator & 11 & 8 & 13 \\
\hline Major outer membrane lipoprotein precursor (OmpF) & 12 & 18 & 9 \\
\hline Hypothetical protein MGC43122 & 13 & 15 & 19 \\
\hline HCV NS5A-transactivated protein 9 & 14 & 14 & 26 \\
\hline KIAA1536 protein & 15 & 13 & 10 \\
\hline Optineurin & 16 & 22 & 15 \\
\hline Hypothetical protein MGC4504 & 17 & 17 & 12 \\
\hline Coiled-coil domain-containing protein 16 & 18 & 16 & 17 \\
\hline RNA-binding protein with multiple splicing & 19 & 29 & 40 \\
\hline Drebrin-like protein & 20 & 11 & 14 \\
\hline 30S Ribosomal subunit protein S15 & 21 & 32 & 28 \\
\hline Bifunctional polymyxin resistance arnA protein & 22 & 23 & 27 \\
\hline Hypothetical protein MGC20255 & 23 & 19 & 35 \\
\hline KIAA1143 & 24 & 20 & 41 \\
\hline Sperm surface protein Sp17 & 25 & 69 & 111 \\
\hline
\end{tabular}

proteins and therefore contribute no information concerning the expression efficiency of the proteins within the ORF pool. In the method presented in this article (Figure 1), proteins purified from a pool of 512 ORFs expressed in $E$. coli are tryptically digested and directly analyzed using LC-MS/MS. From these data, emPAI scores are calculated for each of the identified proteins to obtain a measure of their abundance. The pool of 512 ORFs was expressed and purified in three different experiments using the same conditions, and each of these mixtures was analyzed three times using the sample preparation and LC-MS/ MS method described in the Sample Preparation and MS/MS Analysis section.

The 15 most abundant proteins present within the mixture obtained from the expressed and purified ORFs based on the emPAI values determined in the triplicate analysis of the first ORF pool are shown in Table 1.
As with our previous study that used 2D-PAGE to quantitate the proteins prior to MS identification, SlyD, DnaK, GroEL, and OmpF were among the most abundant $E$. coli proteins. The emPAI values for the top 15 most abundant proteins are given for each of three separate LC/MS analysis. The reproducibility between analyses is very good, resulting in similar emPAI values for each experiment conducted for a single POET pool. Included in this table is the peptide count for each protein identified in the three separate LC/MS/MS experiments. Since the peptide count along with the theoretical peptide count is used to calculate the emPAI values, their reproducibility mirrors that of the corresponding emPAI values.

Three completely separate experiments (i.e., expression, purification, sample preparation, and LC-MS/ MS analysis) were conducted using equivalent aliquots of the 512 ORF-containing POET pool. 


\section{Research Reports}

The mean emPAI values and their associated standard deviations for the 25 most abundant proteins found in the first POET experiment are listed in Table 2. The reproducibility over the three separate experiments is high, and the standard deviations for the mean emPAI values are low. While some minor variances are observed between the mean emPAI values for individual proteins in the three experiments, this discrepancy is well within the limits of the random selection of peptide for sequencing that is seen in data-dependent MS/MS analysis of complex protein mixtures.

Each of the 25 proteins shown in Table 2 was ranked based on their emPAI values calculated from the three separate POET experiments that examined the same pool of 512 human ORFs. As shown in Table 3, proteins that ranked high in one experiment also typically ranked high in the other two experiments. For example, SlyD ranked as either the first or second most abundant protein based on emPAI values in the three POET experiments. Most of the proteins identified within the 10 most abundant proteins in the first experiment were also found within the top 10 of the other two experiments. These results highlight the reproducibility in conducting such studies to find the human clones that express high levels of proteins using this pooled method. One exception was $\mathrm{Sp} 17$, which gave a relatively high emPAI score in the first experiment, but ranked 69 and 11 in experiments two and three, respectively.

The ORFs corresponding to the top 11 emPAI scores were individually subcloned to serve as positive standards for individual expression. In addition, 11 clones that were not identified by LC-MS/MS among the pools of soluble expressed proteins were subcloned to serve as negative controls. Subclones were generated in pDest527, and each of the 22 clones was individually expressed under the same conditions as the POET experiment. Whole-cell extracts were prepared to identify expressed proteins, and soluble fractions were separated by mild detergent lysis. Samples of these fractions were run on SDS-PAGE gels, and the results are shown in Figure 2. All 11 positive clones (as identified by their emPAI values) showed strong expression in the whole-cell extracts, as shown in Table 4. Of these, six showed high levels of expression of soluble protein, while three additional proteins showed moderate levels of expressed soluble protein. Of the remaining two, soluble protein could be detected for one of the proteins using Western blotting analysis with an anti-His6 antibody, and appreciable levels of soluble protein could not be detected for the other.

In the individual validation of 11 negative clones (Table 5), seven showed expression, although as a whole, levels were lower than those observed for the positive clones. Four negative clones showed no detectable expression in Coomassie-stained gels. Only one of the negative proteins was observed in the soluble fraction by Coomassie staining, with a low level of a second protein being detectable using Western blot analysis. The remaining nine negative clones showed no detectable soluble protein. Taken together, these data argue for the strong correlation of high emPAI scores from the soluble POET pool and solubility behavior in individual expression experiments. These data are also similar to the results obtained in our previous study, suggesting that the much more efficient and high-throughput direct LC-MS/ MS approach using emPAI scores is comparable in data quality to the more laborious 2D-PAGE gel approach to POET.

\section{DISCUSSION}

The current trend in biological sciences is to develop methods that collect large amounts of data in a high-throughput manner. These trends can be seen in genome sequencing, messenger RNA (mRNA) array analysis, and proteome characterization by MS. Unfortunately, the optimization of protein expression conditions has generally been limited to trial and error studies in which single clones are tested per experiment (9-11). While automation has enabled multiplexing of these trial

Table 4. Individual Expression Characteristics of 11 Human Clones That Showed High Expression Levels Based on emPAI Values Obtained in POET Experiment

\begin{tabular}{llllll|} 
ORF & ORF ID & emPAl & Expressed? & Soluble? & Figure 2 Gel Lane \\
\hline Protein CGI-38 & BC000691 & 0.456 & Yes & Good & 1 \\
UPF0235 protein C15orf40 & BC019820 & 0.537 & Yes & Good & 2 \\
Programmed cell death protein 5 & BC015519 & 0.436 & Yes & Good & 3 \\
Development pluripotency-4 & BC032846 & 0.267 & Yes & Good & 4 \\
26S Proteasome subunit p27 & BC002383 & 0.230 & Yes & Good & 5 \\
HCV NS5A-transactivated protein-9 & BC007101 & 0.173 & Yes & Fair & 6 \\
KIAA1536 protein & BC003177 & 0.221 & Yes & Fair & 7 \\
Optineurin & BC013876 & 0.166 & Yes & Western & 8 \\
Hypothetical protein MGC4504 & BC019625 & 0.176 & Yes & No & 9 \\
Coiled-coil domain containing 16 & BC011584 & 0.168 & Yes & Fair & 10 \\
Debrin-like protein & BC011667 & 0.199 & Yes & Good & 11 \\
$\begin{array}{l}\text { The final column indicates the lane in the gel shown in Figure 2. ORF, open reading frame; POET, pooled ORF expression technology; emPAI, exponentially modi- } \\
\text { fied protein abundance index. }\end{array}$ & & & & \\
\hline
\end{tabular}




\section{Research Reports}

and error methods, the liquid handling apparatus required to conduct such endeavors is outside of the budget of most laboratories. POET is designed to provide a high-throughput method to simultaneously screen hundreds of ORFs to determine which clones express reasonable amounts of recombinant proteins under a specific set of cloning, expression, and purification conditions. POET is not designed to provide a complete quantitative assessment of every clone in a given pool, as there will undoubtedly be some ORFs that do not express any detectable level of protein. We have previously demonstrated the utility of combining hundreds of ORFs into a single expression system. Identification of clones that produced high levels of recombinant protein was hampered, however, by the need to quantitate by staining 2D-PAGE separated proteins (16). This need directly leads to the major rate-limiting step; coring out each individual protein spot, subjecting it to in-gel digestion, followed by identification using LC-MS/MS. In addition, considerable MS sample preparation and analysis time is wasted on the identification of $E$. coli host proteins, since these proteins are not distinguishable prior to MS analysis. The new, improved method presented in this paper uses a single LC-MS/MS analysis of the entire purified POET pool. Relative abundance is calculated directly by comparing the number of experimentally identified peptides to the number of theoretically observable peptides for each individual protein. This method essentially reduces the time required to identify highly expressing human clones by $1 / n$ (where $n$ is the number of visualized protein spots) compared with the previous method that required visualization of 2D-PAGE separated proteins. Unfortunately, using a single dimension of separation prior to MS analysis limits the number of peptides that can be detected within a given pool. Using multidimensional chromatography, however, not only increases the analysis time but renders the results incompatible with emPAI calculations.

Overall, there is good correlation between an ORFs emPAI value calcu- lated from the LC-MS/MS data and its ability to be expressed in a soluble form. Only one of the 11 clones, hypothetical protein MGC4504, which was predicted to provide a high level of soluble protein based on its emPAI value, failed to do so in the validation study of individual clones. While the reason for this discrepancy is not clear, it should be recognized that pools of $E$. coli cells each containing a different ORF grow in competition with each other. Any slowing of growth caused by expression of an ORF will result in underrepresentation of the corresponding protein in the purified pool. A comparison of the entire list of emPAI values (see Supplementary Table S1 available online at www. BioTechniques.com) shows that the ratio of human to E. coli proteins is approximately 3 -fold higher in the bottom 50 percentile of all proteins that generated an emPAI value (c.f., 6.4 to 2.3). The reason for, or the statistical relevance of, this difference is not obvious. It may be related to the fact that the 512 cloned human ORFs are under the control of an inducible vector, whereas the E. coli proteins are internally regulated based on the needed response by the organism. While E. coli may require a large number of proteins to be expressed for survival under the growth conditions used in this study, low levels of most of these proteins may be sufficient.

One drawback to the POET approach is that multiple proteins are likely being expressed in the same $E$. coli cell, meaning that a protein could potentially be stabilized by an interaction with another ORF. Conditions of higher stress in the POET pool could also lead to upregulation of chaperones, which might also assist in the soluble production of some proteins. The single negative clone that when individually expressed made considerable soluble protein, may be due to a pooling error, as the original POET pools were manually assembled.

The ability to screen a large number of cloned ORFs in a single high-throughput experiment opens up a number of opportunities in structural proteomics. For instance, interesting proteins, such as those classified as hypothetical that have no apparent homology to known proteins, can be prepared in a single pool and their expression efficiency quickly determined. Functionally related pools (e.g., kinases, phosphatase, transcription factors) can also be created, and their recombinant expression tested. POET can also be used in combination with Gateway technology as a filtering tool to determine the best expression vector for individual ORFs. For example, a pool of 500 ORFs could be cloned into multiple different vectors and expressed, purified, and analyzed directly by LC-MS/MS. The emPAI values calculated from each experiment would then indicate the optimal vector for each ORF. The same principle could be used to optimize the expression conditions (e.g., temperature, induction time) for pools of cloned ORFs.

While the quantitation of the protein's relative abundance using a direct LC-MS/MS and emPAI approach represents a major improvement over the previous POET method that required 2D-PAGE/ staining, there are still other areas that can be improved upon. For example, this study analyzed a pool of 512 cloned ORFs, while our previous study analyzed a pool containing 688 . The effect that each recombinant protein may have on the expression of another cannot be easily determined in our study. Therefore, optimization of the number of cloned ORFs that can be most effectively analyzed in a single pool using POET needs to be determined. While conventional thinking is to make the pools as large as possible to increase the number of positive expressors found per experiment, large pools may have an adverse effect on the host machinery, thereby hampering efficiency. Analysis of smaller pools of ORFs may ultimately be a more efficient method, as it may minimize the effect of recombinant protein perturbation on the host cell.

\section{ACKNOWLEDGMENTS}

The authors wish to thank Chacko S. Chakiath and Earl W. Bere III for technical assistance on this project. 
This project has been funded in whole or in part with federal funds from the National Cancer Institute, National Institutes of Health, under Contract NO1-CO-12400. The content of this publication does not necessarily reflect the views or policies of the Department of Health and Human Services, nor does mention of trade names, commercial products, or organization imply endorsement by the U.S. Government. This paper is subject to the NIH Public Access Policy.

\section{COMPETING INTERESTS STATEMENT}

The authors declare no competing interests.

\section{REFERENCES}

1. Collins, J.E., C.L. Wright, C.A. Edwards, M.P. Davis, J.A. Grinham, C.G. Cole, M.E. Goward, B. Aguado, et al. 2004. A genome annotation-driven approach to cloning the human ORFeome. Genome Biol. 5:R84.

2. Lattman, E. 2004. The state of the Protein Structure Initiative. Proteins 54:611-615.

3. Frazier, M., D. Thomassen, A. Patrinos, G. Johnson, C.E. Oliver, and E. Uberhacker. 2003. Stepping up the pace of discovery: the genomes to life program. Proc. IEEE Comput. Soc. Bioinform. Conf. 2:2-9.

4. Levitt, M. 2007. Growth of novel protein structural data. Proc. Natl. Acad. Sci. USA 104:3183-3188.

5. Lundstrom, K. 2006. Structural genomics for membrane proteins. Cell. Mol. Life Sci. 63:2597-2607.

6. Hawkins, T. and D. Kihara. 2007. Function prediction of uncharacterized proteins. J. Bioinform. Comput. Biol. 5:1-30.

7. Mayr, G., F.S. Domingues, and P. Lackner. 2007. Comparative analysis of protein structure alignments. BMC Struct. Biol. 7:50.

8. Kinoshita, K. and H. Nakamura. 2003. Protein informatics towards function identification. Curr. Opin. Struct. Biol. 13:396400.

9. Bursey, E.H., C.Y. Kim, M. Yu, T.C. Terwilliger, and L.W. Hung. 2006. An automated high-throughput screening method for the identification of high-yield, soluble protein variants using cell-free expression and systematic truncation. J. Struct. Funct. Genomics 7:139-147.

10. Chatterjee, D.K. and D. Esposito. 2006. Enhanced soluble protein expression using two new fusion tags. Protein Expr. Purif. 46:122-129.

11. Nallamsetty, S. and D.S. Waugh. 2006. Solubility-enhancing proteins MBP and
NusA play a passive role in the folding of their fusion partners. Protein Expr. Purif. 45:175-182.

12. Haab, B.B., A.G. Paulovich, N.L. Anderson, A.M. Clark, G.J. Downing, H. Hermjakob, J. Labaer, and M. Uhlen. 2006. A reagent resource to identify proteins and peptide of interest for the cancer community: a workshop report. Mol. Cell. Proteomics 5:1996-2007.

13. Stoevesandt, O. and M.J. Taussig. 2007. Affinity reagent resource for human proteome detection: Initiatives and perspectives. Proteomics 7:2738-2750.

14. Qoronfleh, M.W., L.K. Hesterberg, and M.B. Seefeldt. 2007. Confronting highthroughput protein refolding using high pressure and solution screens. Protein Expr. Purif. 55:209-224.

15. Boettner, M., C. Steffens, C. von Mering, P. Bork, U. Stahl, and C. Lang. 2007. Sequence-based factors influencing the expression of heterologous genes in the yeast Pichia pastoris-a comparative view on 79 human genes. J. Biotechnol. 130:1-10.

16. Gillette, W.K., D. Esposito, P.H. Frank, M. Zhou, L.R. Yu, C. Jozwik, X. Zhang, B. McGowan, et al. 2005. Pooled ORF expression technology (POET). Using proteomics to screen pools of open reading frames for protein expression. Mol. Cell. Proteomics 4:1647-1652.

17. Ishihama, Y., Y. Oda, T. Tabata, T. Sato, T. Nagasu, J. Rappsilber, and M. Mann. 2005. Exponentially modified protein abundance index (emPAI) for estimation of absolute protein amount in proteomics by the number of sequenced peptides per protein. Mol. Cell. Proteomics 4:1265-1272.

18. Rual, J.F., T. Hirozane-Kishikawa, T. Hao, N. Bertin, S. Li, A. Dricot, N. Li, J. Rosenberg, et al. 2004. Human ORFeome version 1.1: a platform for reverse proteomics. Genome Res. 14:2128-2135.

Received 10 April 2008; accepted 17 June 2008.

Address correspondence to Timothy D. Veenstra, Laboratory of Proteomics and Analytical Technologies, Advanced Technology Program, SAIC-Frederick, Inc., National Cancer Institute at Frederick, Frederick, MD 21702, USA. e-mail: veenstra@ncifcrf.gov

To purchase reprints of this article, contact:Reprints@BioTechniques.com

\section{sensitivity in pipette control...}

acCu-jet ${ }^{\oplus}$ ro

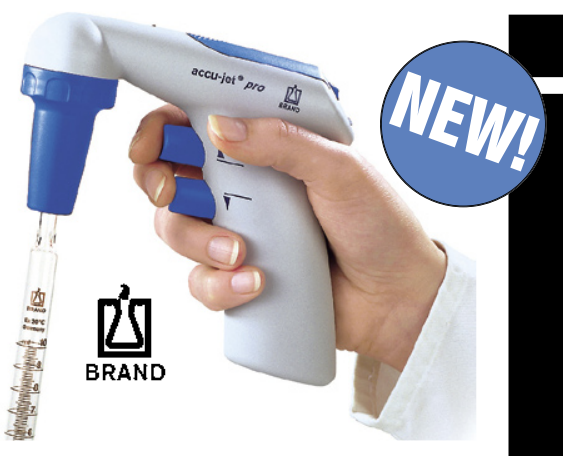

NEW advanced speed control ends meniscus "jumping," even on lowvolume pipettes. And soft blow-out has never been easier!

- NEW selectable maximum speed for ultra-sensitive operations

- Touch-sensitive, continuously variable speed control

- NEW sculpted grip eliminates uncomfortable pressure points

- NEW LED indicator for battery charge status

- Four color accents: dark blue, magenta, forest green, royal blue

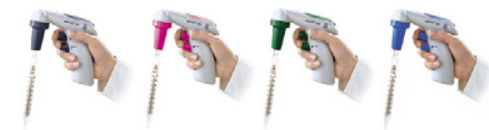

\section{Intro offer -}

\section{Buy 3, get 1 FREE!}

Product and offer details at www.brandtech.com

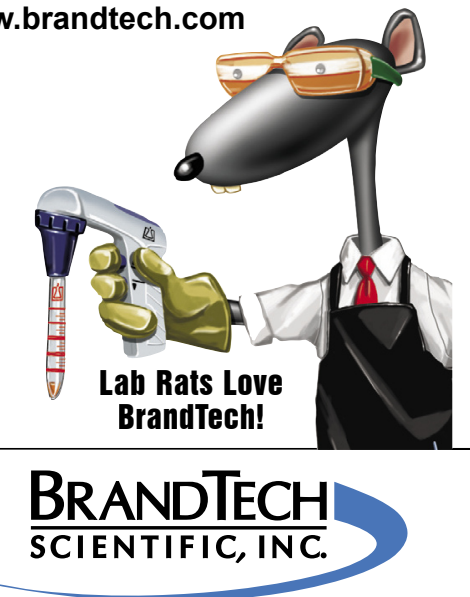

Toll Free (888) 522-2726

www.brandtech.com 